\title{
ASPP2 gets a polarity complex
}

Ankyrin repeats, SH3 domain and proline-rich region containing protein 2 (ASPP2) is a tumour suppressor involved in the regulation of the p53 family. Two groups, Xin Lu, Roberta Sottocornola, Christoph Royer and colleagues, and Shigeo Ohno, Weili Cong, Tomonori Hirose and colleagues, have identified an additional function for this protein - the regulation of cell polarity.

ASPP2 binds to several proteins, indicating that it has functions outside of regulating the p53 family. To investigate further, $\mathrm{Lu}$ and colleagues generated Aspp2 exon 3-knockout mice. In mice of a mixed genetic background, this deletion proved to be lethal. Examination of these embryos identified gross abnormalities in the developing central nervous system (CNS) and disruption of retinal development. Luckily, the same exon 3 deletion had less severe

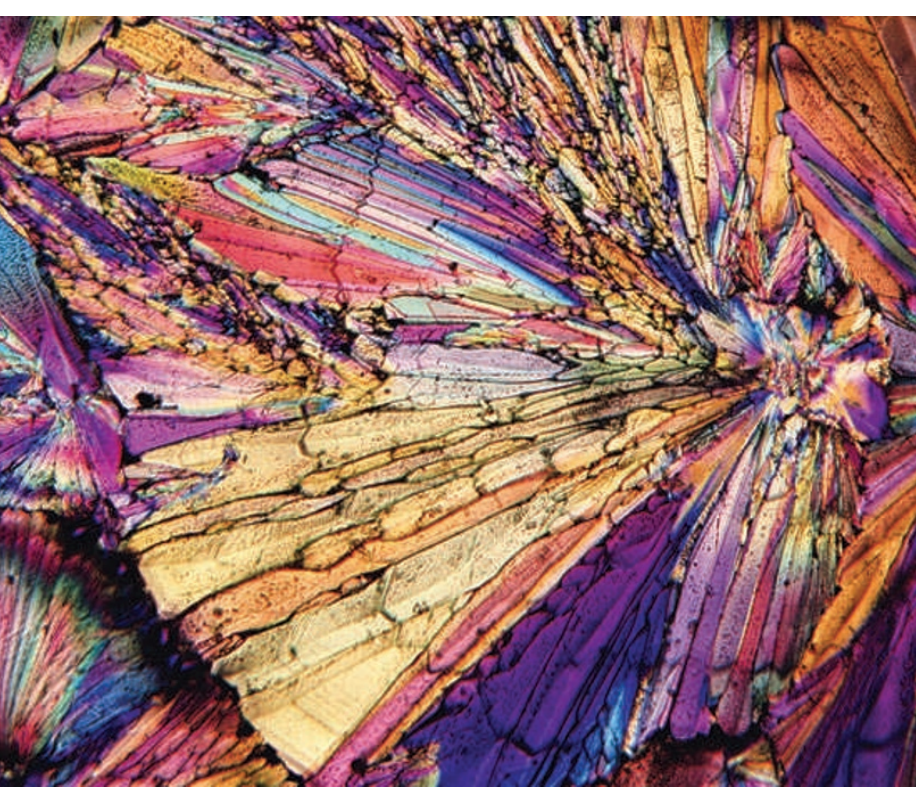

effects in inbred (Balb/c) mice, enabling $\mathrm{Lu}$ and colleagues to examine live young, all of which were born with hydrocephalus and retinal dysplasia.

In situ hybridization in whole mount wild-type embryos showed that Aspp2 mRNA is expressed from embryonic day 9.5 (E9.5) onwards and is highly expressed in the brain, spinal cord and optic cup. Histological analyses of E13.5 Aspp2 exon 3-knockout mice in both genetic backgrounds indicated that the development of the neuroepithelium was disrupted and abnormal proliferation of neural progenitor and migration of differentiating cells was also evident.

A core aspect of highly organised epithelial tissues such as the cerebral cortex is the maintenance of cell polarity. Crucial to this are the apical-junctional complex (AJC) and the Par complex (PAR3-atypicalPKC-PAR6). The authors found that ASPP2 localized with PAR3 and the tight junction protein $\mathrm{ZO} 1$ in wildtype neural epithelial cells, indicating that ASPP2 might function in the maintenance of tight junctions. The strict localization of components of the Par complex and the AJC was disrupted in neural epithelia in Aspp2 exon 3-knockout embryos. Moreover, tight junctions in the choroid plexus, which lines the ventricles and separates the cerebral spinal fluid from the blood, were disrupted in Aspp2 exon 3-knockout mice and this might explain the development of hydrocephalus in these animals.

Further experiments showed that ASPP2 binds PAR3 and is involved in regulating its localization during the initial formation of tight junctions. Indeed, ASPP2 binds to PAR3 at a region of PAR3 that is required for inducing the formation of tight junctions, independently of atypical-PKC and PAR6.

Ohno and colleagues investigated the regulation of the Par complex in epithelial cells by identifying proteins that bound PAR3. ASPP2 co-immunoprecipitated endogenous PAR3, which was bound to atypicalPKC and PAR6. RNA interference experiments indicated that ASPP2 is involved in the formation of tight junctions and the maintenance of apical domains, a feature that is regulated by the Par complex. They also found that the ASPP2-PAR3 interaction is an early event that occurs before atypical-PKC and PAR6 interact with PAR3 in epithelial cells that are establishing polarity. Disruption of PAR3-ASPP2 binding showed the requirement for this interaction in establishing cell polarity.

The results of both of these groups indicate that ASPP2 is a key binding partner of PAR3 that is required for the apical and junctional localization of PAR3, tight junction assembly and apical domain development. This might be the mechanism through which ASPP2 helps to maintain cell polarity and neuroepithelial organization. It will be interesting to determine whether the ability of ASPP2 to regulate cell polarity also impinges on its tumour suppressor activities.

Nicola McCarthy

ORIGINAL RESEARCH PAPERS Sottocornola, R. et al. ASPP 2 binds Par- 3 and controls the polarity and proliferation of neural progenitors during CNS devlopment. Dev. Cell 8 Jul 2010 (doi:10.1016/j.devcel.2010.06.003)| Cong, W. et al. ASPP2 regulates epithelial cell polarity through the PAR complex. Curr. Biol. 8 Jul 2010 (doi: 10.1016/cub.2010.06.024) 